\title{
On the Possibility of Using Water As a Propellant for Powerful Plasma Thrusters
}

\section{Konstantin Gutorov}

National Research Nuclear University MEPhl (Moscow Engineering Physics Institute), Moscow, Russian Federation

\section{Abstract}

The strategy of intensive space exploration (already announced missions of creating a habitable base in the Moon's orbit, lunar bases and flying to Mars) requires the creation of 'space cargo' with a powerful energy source and an electric propulsion system, as well as a sufficient amount of propellant. Achieving megawatt power levels is possible using a nuclear power plant in space and plasma thrusters a kind of plasma accelerators or with high-frequency heating in the presence of a longitudinal magnetic

Corresponding Author: Konstantin Gutorov KMGutorov@mephi.ru Received: 22 July 2018 Accepted: 9 September 2018 Published: 8 October 2018

Publishing services provided by Knowledge E

(c) Konstantin Gutorov. This article is distributed under the terms of the

Attribution License, which permits unrestricted use and redistribution provided that the original author and source are credited.

Selection and Peer-review under the responsibility of the Breakthrough Directions of Scientific Research at MEPhl Conference Committee.
G OPEN ACCESS field. Now, the most proven version is the VASIMR VX-200SS, similar thrusters are being developed in Russia. In the future, the propellant for the plasma engine needs to be replenished directly by the substances available in space, for example, using extensive water supplies. There are some encouraging results on the use of water as a propellant for powerful plasma thrusters - these are experiments with microwave heating, where the efficiency gain is about $20 \%$ compared to argon, and theoretical work on the helicon discharge, which shows that the efficiency comparable to argon should be achieved at a plasma temperature above $5 \mathrm{eV}$. Now, direct experimental confirmation of the efficiency of the helicon discharge in water vapor is required.

Keywords: plasma thruster, water, helicon discharge, auto-oscillations

\section{Introduction}

Space exploration is an inevitable process in the development of mankind, which has significantly accelerated at the present time through the participation of private companies. The total volume of the space industry market exceeded $\$ 300$ billion and continues to grow; competition in this market is increasing. The need for powerful 'space cargo' and possible scenarios for their application are described in many articles, for example [1-3]. The tasks that are being set now are the creation of an inhabited base in the Moon's orbit, the creation of lunar bases, and the flight to Mars. Already at this stage, it may be advisable to use the water available in the form of ice on the Moon. The capabilities of well-designed ion or stationary plasma thrusters in combination 
with solar batteries are limited to a few tens of kilowatts. At present, flight variants of ion and Hall plasma thrusters with a power of about $5 \mathrm{~kW}$ are quite well tested and thruster models for 35-50 kW are being investigated, which, apparently, is the power limit for one module $[4,5]$. To provide a megawatt power level, it is proposed to use a large number of thrusters with a power of tens of kilowatts (well-tested structures working on xenon) or the development of other types of thrusters with higher power, and using other working substances - argon, lithium, iodine.

It is possible to reach new power levels (up to a megawatt) using a nuclear power plant in space, a project for the creation of which is currently being implemented in Russia, and plasma thrusters such as plasma accelerators or high-frequency heating in systems with a longitudinal magnetic field. Now, the most proven alternative is an electrodeless VASIMR thruster with the creation of plasma in a helicon source and its subsequent heating by the ion cyclotron heating method. At present, ground-based tests of the flight modification of the VASIMR VX-200SS thruster operating on argon with a total power of $100 \mathrm{~kW}$ in a pulse of $10 \mathrm{~s}$ have been carried out. The next stage of the tests is work with a total power of $100 \mathrm{~kW}$ for 10 hours and the final stage - a demonstration of continuous operation at 100 hours is planned for 2018 [6]. Plasma thrusters with high-frequency heating are being developed in Russia in Novosibirsk at the Budker Institute of Nuclear Physics [7] and in Moscow in the Research Center 'Kurchatov Institute' [8]. Optimization of such thrusters for operation with water propellant will allow to refuse delivery of a large amount of argon or xenon from the Earth to replenish fuel resources. Data on the presence of water in solid form in the solar system are not currently questioned, for example, an analysis of the amount available for use is given in [9].

\section{Approaches and Methods}

The main approach in the design of powerful plasma thrusters with a long service life is the use of electrodeless discharges and methods of plasma heating, as well as the use of a strong magnetic field for thermal insulation of plasma. The application of these approaches makes it possible to minimize the contact of the plasma with the surface of the thruster elements, and, consequently, to reduce their erosion. In practice, there are usually several stages in the formation of the plasma flow. The first stage is the creation of a dense plasma, for which a highly effective helical discharge with an external volumetric antenna is usually used. The second stage is the additional heating of ions by the radiofrequency waves at the ion cyclotron resonance and the formation 
of their directed flow. Experimental studies on the use of water as a working fluid for high-power plasma thrusters with high-frequency heating are conducted in the USA $[10,11]$. In these studies, it is shown that the use of water gives a gain in efficiency of about $20 \%$ in comparison with argon. Modeling of processes in electrodeless helicon discharge on water vapor and comparison with discharge in argon is given in [12], where it is shown that the increase in the energy cost of an ion for water vapor is practically leveled at a plasma temperature of more than $5 \mathrm{eV}$. At the current time, it is required to determine experimentally the optimal regimes for helicon discharge on water vapor in a longitudinal magnetic field for use as the first stage of a powerful plasma thruster, which will allow further optimization of the ion cyclotron heating stage.

\section{Results and Discussion}

We chose a special type of discharge for testing the discharge conditions for water vapor, with self-excitation of helicon waves in a plasma-filled resonator, which does not require matching systems and high-frequency power supply. The experiments were carried out in the plasma facility PR-2, the scheme of which is shown in Figure 1. A detailed description of the main operating modes and diagnostic systems of the facility is given in [13]. The device is an open magnetic trap with an electron gun, working in beam-plasma generator regime. Helicons can be excited inside a special module, which is sealed at the end opposite to the gun. The sealing is movable by almost the full module length $(0.8 \mathrm{~m})$. The module has its own magnetic coil wound around the resonator tube $\varnothing 50 \mathrm{~mm}$. The coil is confined by the $\varnothing 100 \mathrm{~mm}$ outer tube welded with the inner one.

The copper winding tape and tubes are cooled by water flowing along the gap between tubes. It permits us to vary magnetic field in wide range - up to $0.12 \mathrm{~T}$ and remove thermal load from plasma - up to $3 \mathrm{~kW}$ stationary. The module outer end is closed by a changeable water-cooled plate sealed to electrode assembly welded to ceramic feedthroughs. The module inner end is opened into the main chamber. The diagnostics include movable Langmuir and magnetic probes, high frequency voltage dividers, Rogovski coils, mass analyzers, spectrometers. Special probes and targets can be also introduced into plasma flows through a sluiceway.

The auto-oscillating regime has been obtained after $\mathrm{Ar}^{+}$cleaning and following formation on the plate surface (by deposition or oxidation) of a thin dielectric layer necessary for appearing of the unstable plasma-surface contact. The beam-plasma 


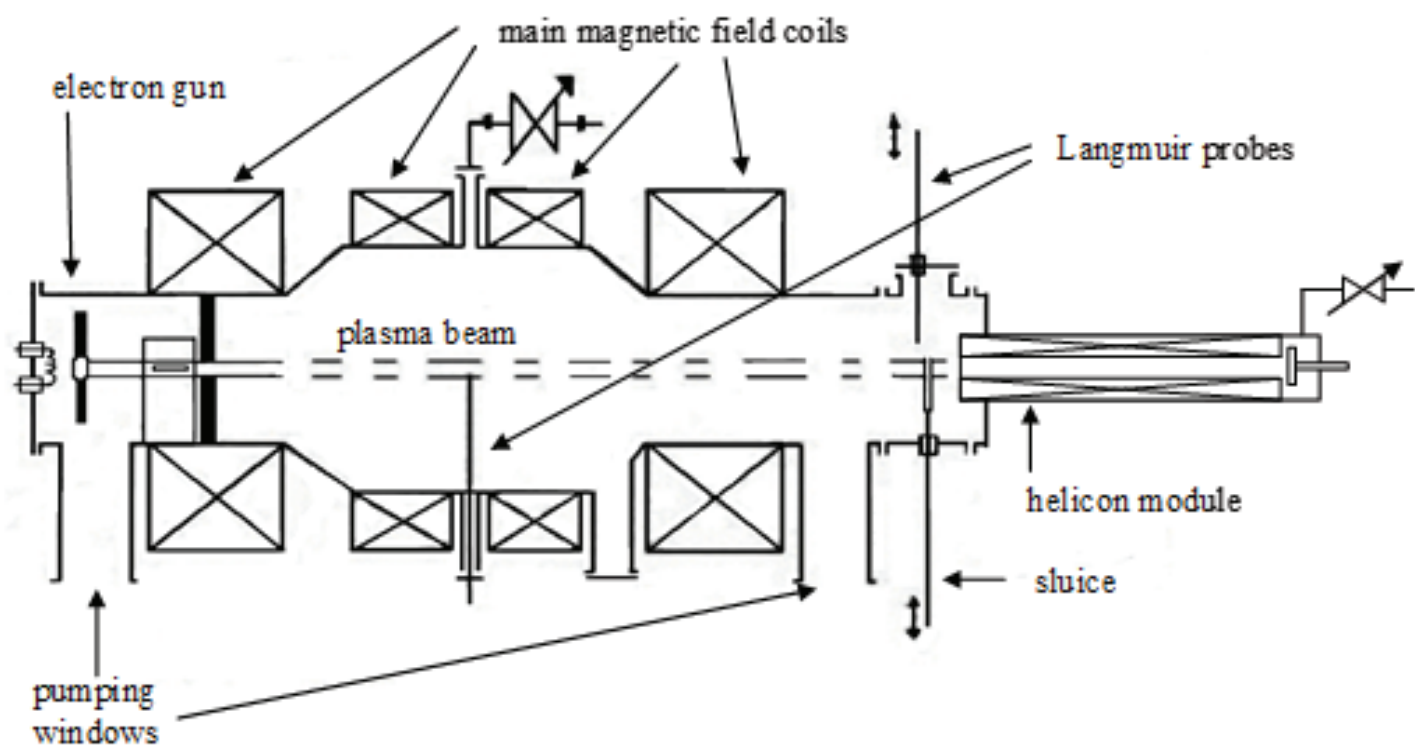

Figure 1: Experimental facility scheme.

discharge in hydrogen at pressure in the range of $10^{-2}-10^{-1}$ Pa provides the initial filling of the module with plasma (of about $10^{11} \mathrm{~cm}^{-3}$ density) and fast electrons. Hydrogen is most convenient due to the low sputtering of the dielectric layer, which can exist in this case stably. The primary plasma channel is much thinner (10 $\mathrm{mm}$ ) than the module inner tube $(50 \mathrm{~mm})$. With rising of a negative $D C$ voltage bias of the plate, a monotonous current increase was observed with following transition of a stable interaction to a very unstable one at the threshold voltage value of $100-200 \mathrm{~V}$ depending on primary electron beam parameters. The amplitude of oscillations with the eigenfrequency ( $\sim 50 \mathrm{MHz}$ ) markedly increases with bias voltage.

In the auto-oscillating mode, the alternating electric fields and power dissipation in plasma increase significantly. The power load from DC supply, plasma channel width, plasma density and ion output also increase manifold. After onset, the auto-oscillating discharge can exist even without primary electron beam. The amplitude of current oscillations exceeds the constant bias supply current by about one order of magnitude, which after auto-oscillations onset increases manifold. It points onto the rather high $Q$ factor of resonator. This statement is consistent with relatively narrow peaks in Fourier spectrum, so $Q$-factor can be estimated as 50-100.

The module acts like a resonator for the wave excitation, while the negatively biased electrode plate acts like an amplifier. An analysis shows that helicon waves could arise and be amplified in this system. In traditional helicon sources, $m=+1$ mode is excited due to the antennae requirements, while $m=0$ mode is excited in presented system due to the rotational symmetry. It is an advantage of this system because the 
power deposition peak occurs at the axis. This allows obtaining more intensive and homogenous plasma beam.

The structure of the generated right-hand polarized helicon wave is shown in Figure 2. The wave is electrostatic near the electrodes end purely electromagnetic near the end of the open resonator or in the center of the closed resonator (resonator could be closed by a target or a diaphragm).

Boundary condition for conducting wall (zero poloidal electric field, $E_{\varphi}=0$ or zero radial magnetic field, $B_{r}=0$ ) and for a dielectric wall (zero radial current, $j_{r}=0$ ) is the same. Low damping of helicon waves allows power channeling along magnetic field to significant distances from plasma generator, so wave dumping compensate particle and energy losses. This effect allows separating the plasma-generation zone and ion-heating zone.

A plasma-electrode contact is the most important for the wave excitation. Only normal to the surface electric field can exist near the conducting plate, so $E_{r}$ and $E_{\varphi}$ should be zero, but this could not be achieved simultaneously. The presence of the weakly conducting sheath with a space charge between plasma and an electrode solves this problem. A radial electrostatic field connected with the space charge can exist near the sheath border. The azimuthal electric field can exist only at some distance from electrodes.

The biased electrode with the high secondary electron-electron emission coefficient can amplify current perturbation at the presence of non-equilibrium fast electrons. Due to the high yield of secondary electrons, the local positive space charge near the electrode will be decreased causing a radial electrostatic field and the further current growth. The radial electrostatic field $E_{r}$ and the axial magnetic field $B_{0}$ evoke an azimuthal electron drift $j_{\varphi}$ and an alternating magnetic field $B_{\varphi}$.

This self-oscillating discharge regime was obtained for various electrode materials (aluminum, tungsten, tantalum, stainless steel with a silicon oxide film) for hydrogen, deuterium and oxygen as working gases. The use of a water vapor source with the required capacity, as was done in [14], will make it possible to investigate in such a system a helical discharge in water vapor.

\section{Conclusion}

New high-efficient type of discharge - 'auto-oscillating helicon discharge' was investigated. Auto-excitation of helicon-type standing waves is used for the producing of the new low-pressure discharge and generation of intensive and highly ionized plasma 


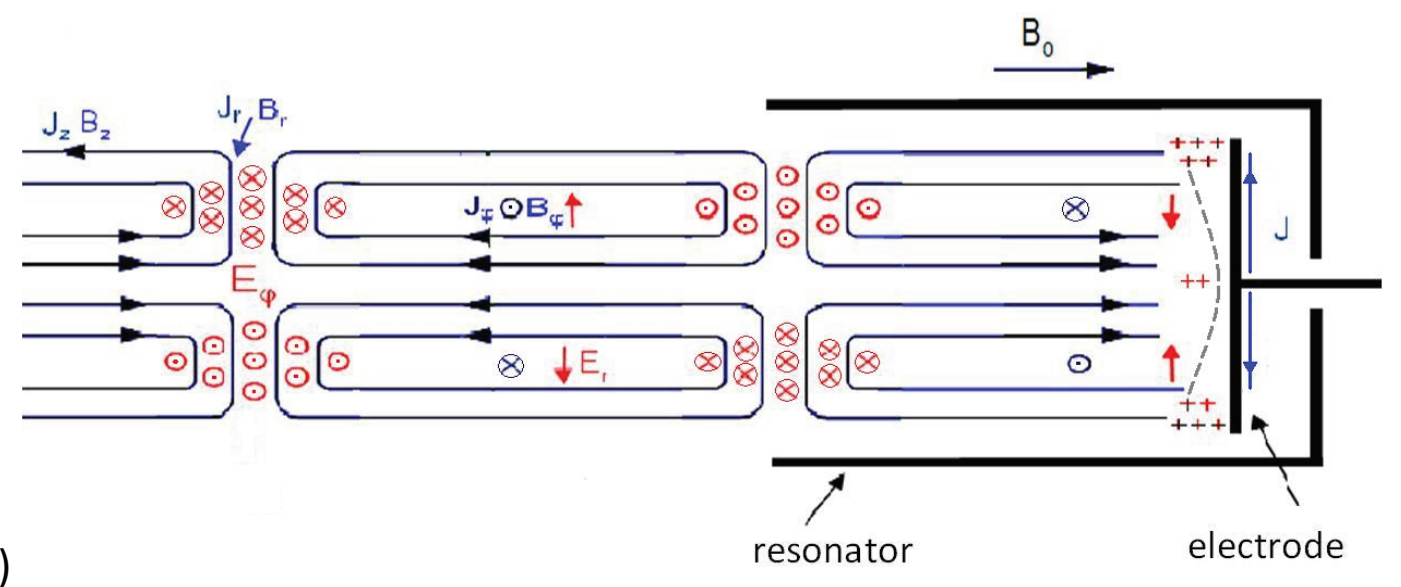

(a)

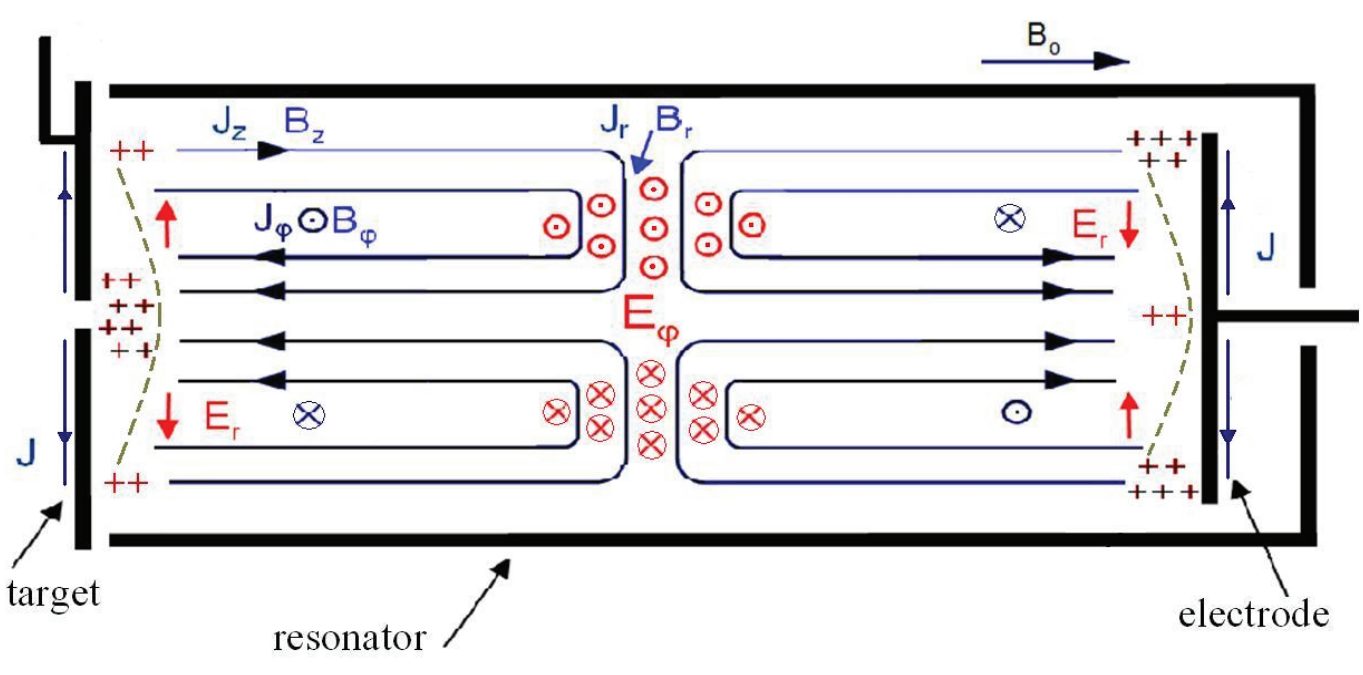

Figure 2: The wave structure: (a) - open resonator; (b) - closed resonator.

flows. The corresponding regimes need only DC power supplies, RF electromagnetic oscillations can be confined within the volume of resonator. The presence in plasma of alternating electric fields and currents determines the enhanced power efficiency. This discharge is similar in its characteristics to the traditional helicon discharge excited by antennas and will be used to test the operating modes of the first stage of a powerful electrodeless plasma thruster.

\section{References}

[1] Koroteev, A. A., Akimov, V. N., and Popov, S. A. (2011). The flight, vol. 4, pp. 93-99 (in Russian).

[2] Kosenko, A. B. and Sinyavsky V. V. (2013). Space Engineering and Technology, vol. 2, pp. 72-84 (in Russian). 
[3] Kuvshinova, E. Yu., Akimov, V. N., Arkhangelsky, N. I., et al. (2016). Space Engineering and Technology, vol. 3, no. 14, pp. 62-70 (in Russian).

[4] Boykachev, V. N., Gusev, Yu. G., Zhasan, V. S., et al. (2014). Space Engineering and Technology, vol. 1, no. 4, pp. 48-59 (in Russian).

[5] Khamits, I. I., Filippov, I. M., Burylov, L. S., et al. (2017). Space Engineering and Technology, vol. 1, no. 16, pp. 32-40 (in Russian).

[6] Giambusso, M., Carter, M. D., Squire, J. P., et al. (2017). Progress in the VASIMR ${ }^{\circledR}$ VX200SS plasma testing program, in 53rd AIAA/SAE/ASEE Joint Propulsion Conference, 2017.

[7] Beklemishev, A. D. (2016). Design optimization of a helical plasma thruster. AIP Conference Proceedings, vol. 1771, article no. 070007.

[8] Novikov, M. S., Zhiltsov, V. A., Kulygin, V. M., et al. (2015). Scientific readings on aviation, dedicated to the memory of N.E. Zhukovsky, no. 3, p. 131 (in Russian).

[9] Bagrov, A. V. (2016). Materials of the round table "Creation of small space vehicles. Actual problems and ways to solve them", pp. 56-68 (in Russian).

[10] Ziemer, J. and Petr. R. (2002). 38th AIAA Joint Propulsion Conference, AIAA-2002-4273.

[11] Brandenburg, J. E., Kline, J., and Sullivan, D. (2005). IEEE Transactions on Plasma Science, vol. 33, no. 2, pp. 776-782.

[12] Petro, E. M. and Sedwick, R. J. (2017). Journal of Propulsion and Power. DOI: $10.2514 / 1$. B36389

[13] Kurnaev, V., Vizgalov, I., Gutorov, K., et al. (2015). Journal of Nuclear Materials, vol. 463, article no. 48808, pp. 228-232.

[14] Sorokin, I. A., Vizgalov, I. V., Gutorov, K. M., et al. (2015). Bulletin of the Lebedev Physics Institute, vol. 42, no. 12, pp. 350-355. 